\title{
Milling Chatter Prediction Based on the Information Entropy and Support Vector Machine
}

\author{
Chen Bing ${ }^{1}$, Yang Jie ${ }^{1}$, Zhao $\mathrm{Ju}^{1}$, Ren Jingbo ${ }^{1}$ \\ ${ }^{1}$ Key Laboratory of Contemporary Design and Integrated Manufacturing Technology, Ministry of \\ Education, Northwestern Polytechnical University, Shannxi Xi'an, 710072, China
}

Keywords: Milling Chatter Prediction, Multi-scale Permutation Entropy, Wavelet Packet Energy entropy, Support Vector Machine

Abstract.This paper proposes a method based on information entropy and support vector machine predict chatter in milling, it uses multi-scale permutation entropy and wavelet packet energy as the milling chatter premonition features, we select parameters of these identifying features by experimental analysis, and predict chatter using the SVM which use these two identifying features as its input. The results show that this method can effectively predict the occurrence of milling chatter, correct rate is $95.8 \%$.

\section{Introduction}

There are many studies on milling chatter which was launched around the feature recognition and classification. In this case, the milling chatter has occurred and the chatter grain has been generated on the surface, so in order to improve the accuracy and workpiece quality, we need to research the methods to predict milling chatter. As long as we identify the premonition of milling chatter, we have enough time to send a response signal to milling chatter control system, and take the appropriate control measures to prevent the occurrence of milling chatter.

Milling chatter premonition monitoring based on selecting the appropriate chatter premonition feature and classification rules. In terms of feature selection, the milling force signal increases sharply from the perspective of the time domain, meanwhile, from the perspective of the frequency domain, it generated chatter frequency. Both the permutation entropy ${ }^{[1]}$ and wavelet packet energy entropy of milling force signal we study in this paper can monitor the milling chatter symptom effectively from the perspective of the time domain and frequency domain. In terms of decision-making, SVM is a machine learning method based on statistical learning theory, it can solve the high dimensional pattern identify issues which are nonlinear, local extremes and the small sample, we will use SVM as the classifier to predict milling chatter premonition.

\section{Selection and Extraction of Chatter Premonition Monitoring Feature}

Assuming a time series $X=\{x(i), i=1,2, \ldots, n\}$, we can obtain a matrix ${ }^{[2],[3]}$ by n-dimensional space reconstruction of $X$

$$
\left[\begin{array}{cccc}
x(1) & x(1+\tau) & \cdots & x(1+(m-1) \tau) \\
x(2) & x(2+\tau) & \cdots & x(2+(m-1) \tau) \\
x(j) & x(j+\tau) & \cdots & x(j+(m-1) \tau) \\
\cdots & \cdots & \cdots & \cdots \\
x(K) & x(K+\tau) & \cdots & x(K+(m-1) \tau)
\end{array}\right]
$$

There are $m$ ! sequences of different permutations when reconstruction of the sort for each component, permutation entropy is defined as

$$
H_{P}(m)=\sum_{j=1}^{m !} P_{i} \ln P_{i}
$$

Normalize the $H_{P}(m)$. 


$$
H_{P}=H_{P}(m) / \ln (m !)
$$

Permutation entropy can change into different scales of time series by introduce the scale factor $s$,

$$
y^{s}(j)=\frac{1}{S} \sum_{i=(j-1) s+1}^{j s} x(i)
$$

According to the above arrangement entropy algorithm, we can compute permutation entropy at different scales of time series, that is, multi-scale permutation entropy.

The definition of wavelet packet energy entropy is,

$$
\left\{\begin{array}{c}
T=-\sum_{i=1}^{n} p_{i} \cdot \log p_{i} \\
p_{i}=E_{i} / \sum_{i=1}^{n} E_{i}
\end{array}\right.
$$

In the type, $n=2^{j}, j$ is the layer of wavelet packet decomposition.

\section{Feature Classification Based on SVM}

The SVM's goal is to find an optimal hyperplane which can makes the classification interval maximum $^{[4]}$. Here is a training set $\left\{\left(x_{i}, y_{i}\right), i=1,2, \ldots n\right\}$ whose samples number is $N, y_{i} \in\{-1,1\} \quad x_{i} \in R^{n}$ is the sample index set, the optimal hyperplane equation is $w \cdot x+b=0, H_{1}: w \cdot x+b=-1$ and $H_{2}: w \cdot x+b=1$ are two plane Parallel to it. Then, the distance $\gamma$ between the plane $H_{1}$ and $H_{2}$ can be expressed as

$$
\gamma=2 \times \frac{|g(x)|}{\|w\|}=\frac{2}{\|w\|}
$$

For linear inseparable classification problems, this problem can be expressed as follows by introducing slack variables $\xi_{i}$, penalty factor $C$, lagrange multipliers $\alpha_{i} \geq 0$ and $\beta_{i} \geq 0$, then, construct Lagrange function, problem above can be expressed as the following questions,

$$
\left\{\begin{array}{c}
\max Q(\alpha)=\sum_{i=1}^{n} \alpha_{i}-\frac{1}{2} \sum_{i=1}^{n} \sum_{j=1}^{n} \alpha_{i} \alpha_{j} y_{i} y_{j}\left(x_{i} \cdot x_{j}\right) \\
\text { s.t. } \quad \sum_{i=1}^{n} y_{i} \alpha_{i}=0 \\
C \geq \alpha_{i} \geq 0, i=1,2, \ldots, n
\end{array}\right.
$$

In order to make the training sample linearly separable, SVM change the input space into high dimensional feature space by introducing the kernel function. We choose the Gaussian radial basis kernel function as kernel function in this paper,

$$
K\left(x_{i}, x\right)=\exp \left\{-\frac{\left|x_{i}-x_{j}\right|^{2}}{2 \sigma^{2}}\right\}
$$

Replace the $\left(x_{i}, x_{j}\right)$ by $K\left(x_{i}, x_{j}\right)$, we get the following optimization problem,

$$
\left\{\begin{array}{c}
\max Q(\alpha)=\sum_{i=1}^{n} \alpha_{i}-\frac{1}{2} \sum_{i=1}^{n} \sum_{j=1}^{n} \alpha_{i} \alpha_{j} y_{i} y_{j} K\left(x_{i}, x_{j}\right) \\
\text { s.t. } \sum_{i=1}^{n} \alpha_{i} y_{i}=0 \alpha_{i} \geq 0(i=1,2, \ldots, n)
\end{array}\right.
$$

Now the classification function equation is 


$$
g(x)=\operatorname{sgn}\left[\sum_{i=1}^{n} \alpha_{i}^{*} y_{i} K\left(x_{i}, x\right)+b^{*}\right]
$$

\section{Milling Chatter Prediction Model and Process}

Milling chatter prediction method based on information entropy and support vector machine can been expressed by the following processes, prediction model is shown in Figure 1.

(1) Milling force signal de-noising;

(2) Divide the timing data of milling force with the data length of 128 non-overlapping windows, then, calculate the multi-scale permutation entropy of each sliding window in different scales, choose the multi-scale permutation entropy which distinct various milling stages clearest as feature $T_{1}$.

(3) Decompose milling force signal with wavelet packet and calculate the wavelet packet energy entropy of each band as the feature $T_{2}$.

(4) Determine whether be in the milling state or not by the permutation entropy.

(5) Get the feature vector $\left[T_{1}, T_{2}\right]$ as the SVM input values, train the SVM by the training samples, then, verify if the SVM can accurately determine different milling status by test samples.

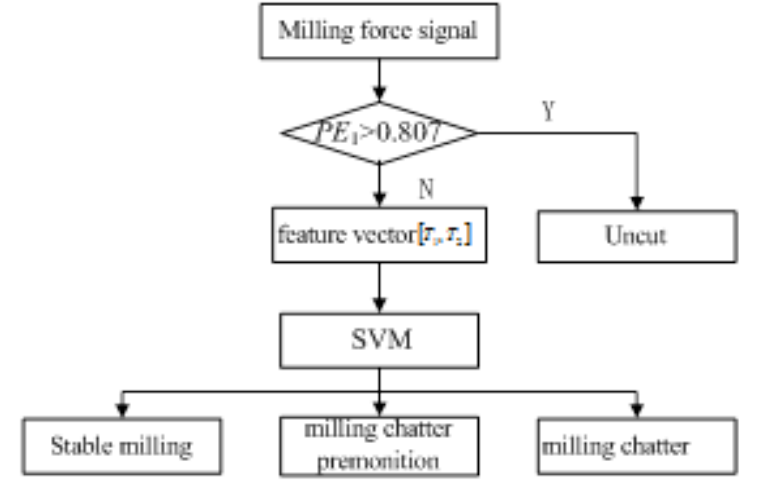

Fig. 1 The milling chatter prediction model

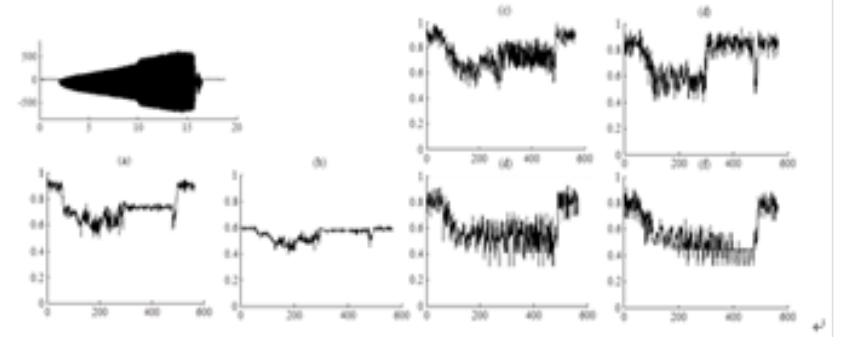

Fig.2 The scale 1-6 permutation entropy of milling force

\section{Experimental Verification and Analysis}

The experimental device in this paper: JOHNFORD VMC-850 four-axis vertical machining centers, Kistler 9123C dynamic rotating dynamometer, Kistler RCD5223 charge amplifier, 5697A data acquisition card, computer and software DynoWare 2.5.3.8.

In this paper, we choose the aluminum alloy 7075 as experimental material. Spindle speed is taken as $n=1850 \mathrm{r} / \mathrm{min}$, the feed rate is taken as $F=420 \mathrm{~mm} / \mathrm{min}$, the axial depth of cut is taken as $a_{p}=11 \mathrm{~mm}$, and the radial depth of cut varies from $0 \mathrm{~mm}$ to $3 \mathrm{~mm}$. Tool wear has a direct impact on the milling force, So using 4F VHSS-AI normal wear superhard straight shank end mill in our experiment. Because the sampling frequency of milling force signal is $3840 \mathrm{~Hz}$, we choose ${ }^{[1]}$ the data length for $w=128$, the embedding dimension for $m=4$ and the delay times for $\tau=1$. The milling force recorded by rotary dynamometer and its corresponding different scales permutation entropy were shown in Figure 2.

Figure 2 (a)-(f) represent the scale 1-6 permutation entropy. As is shown in figure 2 (a), the permutation entropy values is close to 0.92 when the tool didn't involve in cutting. When entering the milling state, the permutation entropy values drastically reduced, but the change of permutation entropy between stable milling and milling chatter isn't clear. However, Figure 2 (d) which represent the scale 4 can distinguish these two milling state easily. So we choose the scale 1 to determine whether the tool participate in milling, and take the scale 4 as the chatter premonition 
monitoring feature $T_{1}$.

Tab.1 Milling chatter premonition monitoring test data.

\begin{tabular}{|c|c|c|c|c|c|c|c|}
\hline No. & $\begin{array}{l}\text { Spindle } \\
\text { spedt } \\
\text { (rpm). }\end{array}$ & $\begin{array}{l}\text { Feod rate } \\
\text { (mm min) }\end{array}$ & $\begin{array}{l}\text { Radial } \\
\text { depth of } \\
\text { cut (mm). }\end{array}$ & $\begin{array}{l}\text { Axial depth } \\
\text { of cut (mm). }\end{array}$ & $\begin{array}{l}\text { Output } \\
\text { cunting } \\
\text { stave. }\end{array}$ & $\begin{array}{l}\text { Target of } \\
\text { cunng } \\
\text { wate. }\end{array}$ & $\begin{array}{l}\text { The } \\
\text { results. }\end{array}$ \\
\hline 1. & 1900. & 4220. & 3.0 . & $11.0 x$ & 1. & 1. & 1. \\
\hline 2. & 2000 . & 420. & 2.8. & 11.0 . & 1. & i. & 1. \\
\hline 3. & 2350 . & 400 . & 3.0 . & 11.0 . & 1. & 1. & 1. \\
\hline 4. & 2400 . & 400. & 3.2 . & 10.0 . & 1. & 1. & 1. \\
\hline 5. & 2500 . & 400. & 3.3 . & 10.0 . & 1. & 1. & 1. \\
\hline 70. & 1850 . & 420 . & 20 . & 11.4 & 3. & 3. & 1. \\
\hline 71. & 3000 . & 360. & 28. & 12.0 . & 3. & 3. & 1. \\
\hline 72. & 3100. & 340. & 2.8 . & 11.8. & 3 . & 3. & i. \\
\hline 73. & 3150. & 340. & 2.9. & 12.0 . & 3. & 3. & 1. \\
\hline 74. & 2200 . & 420. & 3.2. & 12.0 . & 3. & 3. & 1. \\
\hline 75. & 3050 . & 420 . & 2.7. & $12.0 \mathrm{~N}$ & 3. & 3. & 1. \\
\hline
\end{tabular}

Do 75 experiments as table 1, each of them is the different combinations of spindle speed, feed rate, radial and axial depth of cutting, numbers 1, 2, and 3 respect Stable milling, milling chatter premonition and milling chatter. Do 25 experiments of each milling state, 17 groups are taken as training sample, 8 groups are taken as test sample. Here are only 10 sets of data for milling test process parameters in table 1because of the limited space in this paper. Then, We calculated the 3-6 layers db2 wavelet packet energy entropy as is shown in Figure3.

According to the results in Figure3, 3 layers wavelet packet decomposition of wavelet packet energy entropy has smallest changes in the amplitude of chatter, what's more, the more layers, the more time-consuming to calculate. Therefore, we choose the 3 layers wavelet packet decomposition of wavelet packet energy entropy as the chatter premonition monitoring feature $T_{2}$.

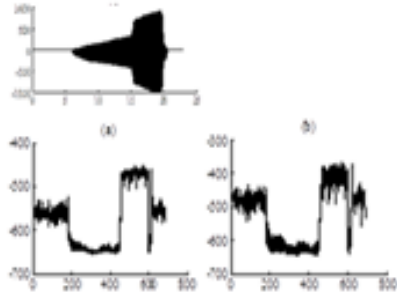

Fig.3 The 3-6 layers db2 wavelet packet energy entropy

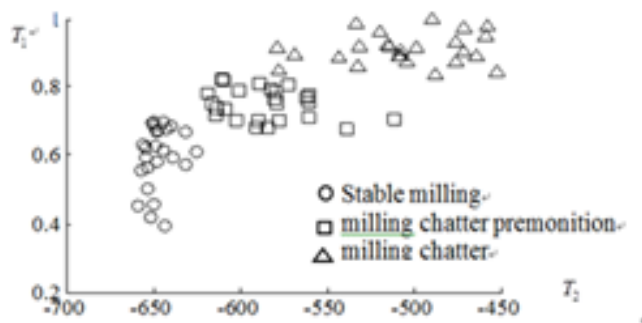

Fig.4 The spatial distribution of feature vector $\left[T_{1}, T_{2}\right]$

Calculate the scale 4 permutation entropy $T_{1}$ and 3 layers wavelet packet energy entropy $T_{2}$ which are the feature vectors of 17 groups of samples for each milling state, get the feature vector $\left[T_{1}, T_{2}\right]$ as the SVM input value, train SVM by the training sample. Finally, verify the effectiveness of SVM by 24 groups of test sample. Experimental results show that the milling chatter prediction system can effectively identify the milling chatter premonition, accuracy was $95.8 \%$, the algorithm computation time is $0.029 \mathrm{~s}$.

\section{Conclusion}

The milling chatter prediction method based on the Information Entropy and Support Vector Machine we proposed in this paper can identify the milling chatter premonition effectively, it reached the purpose of milling chatter prediction, and the prediction accuracy rate is about 95.8\%. The method is simple and intuitive, fast in calculation speed, meanwhile, it is suitable for small sample classification recognition with high accuracy, so it is in line with the needs of practical application, and has engineering application value.

\section{Acknowledgement}

In this paper, the research was sponsored by the 973 program “ intelligent manufacturing basic research of complex and high quality parts” 2-- "evolutionary models of complex thin-walled parts machining processes and adaptive evolutionary mechanism." 


\section{References}

[1] Gradišek J, Govekar E, Grabec I. Using coarse-grained entropy rate to detect chatter in cutting[J]. Journal of sound and vibration, 1998, 214(5): 941-952.

[2] Bandt C, Pompe B. Permutation entropy: a natural complexity measure for time series[J]. Physical Review Letters, 2002, 88(17): 174102.

[3] Takens F. Detecting strange attractors in turbulence[M]. Dynamical systems and turbulence, Warwick 1980. Springer Berlin Heidelberg, 1981: 366-381

[4] Shao Q, Feng C J. Pattern Recognition of Chatter Gestation Based on Hybrid PCA-SVM[J]. Applied Mechanics and Materials, 2012, 120: 190-194.

[5] Cao Y, Tung W, Gao J B, et al. Detecting dynamical changes in time series using the permutation entropy[J]. PHYSICAL REVIEW-SERIES E-, 2004, 70(4; PART 2): 046217-046217. 